\title{
Erratum: Polarized beam conditioning in plasma based acceleration [Phys. Rev. ST Accel. Beams 14, 071303 (2011)]
}

\author{
J. Vieira, C.-K. Huang, W. B. Mori, and L. O. Silva \\ (Received 18 August 2011; published 20 September 2011) \\ DOI: 10.1103/PhysRevSTAB.14.099901 \\ PACS numbers: 52.38.Kd, 41.75.Lx, 41.75.Ht, 52.65.Rr, 99.10.Cd
}

Equation (2) in paper [1] should read [2]

$$
\frac{d \mathbf{s}}{d t}=-\left[\left(a+\frac{1}{\gamma}\right) \mathbf{B}-\left(a+\frac{1}{1+\gamma}\right) \mathbf{v} \times \mathbf{E}-\mathbf{v} \frac{a \gamma}{\gamma+1} \mathbf{v} \cdot \mathbf{B}\right] \times \mathbf{s}=\mathbf{\Omega} \times \mathbf{s},
$$

where $\Omega$ is $\boldsymbol{\Omega}=[(a+1 / \gamma) \mathbf{B}-[a+1 /(\gamma+1)] \mathbf{v} \times \mathbf{E}-\mathbf{v} a \gamma \mathbf{v} \cdot \mathbf{B} /(\gamma+1)]$. The spin precession of ultrarelativistic electrons, examined in [1], is well described by Eq. (2) from Ref. [1], and can be readily obtained through Eq. (1) by taking $\gamma \gg 1$ such that $1 /(\gamma+1) \approx 1 / \gamma$.

In addition, a more rigorous value for the anomalous magnetic moment of the electron $a$, which is defined in the paper as $a \approx 0.0011614$, is given by $a=1.15965218076 \times 10^{-3}$ [3].

We note that the derivation and conclusions presented in the paper are not affected by these changes.

The authors would like to thank an interested reader of the paper for pointing out these corrections.

[1] J. Vieira, C.-K. Huang, W. B. Mori, and L. O. Silva, Phys. Rev. ST Accel. Beams 14, 071303 (2011).

[2] S. R. Mane, Y. M. Shatunov, and K. Yokoya, Rep. Prog. Phys. 68, 1997 (2005).

[3] National Institute of Standards and Technology (NIST) reference on Constants, Units, and Uncertainty [http://physics.nist.gov/ cuu/Constants].

Published by the American Physical Society under the terms of the Creative Commons Attribution 3.0 License. Further distribution of this work must maintain attribution to the author(s) and the published article's title, journal citation, and DOI. 